\title{
An Approach to the Performance-Oriented Model of Variable-Speed Wind Turbines
}

\author{
Alejandro Rolán ${ }^{1}$, Student Member, IEEE, Álvaro Luna, Joan Rocabert, Student Member, IEEE, \\ Daniel Aguilar, and Gerardo Vázquez, Student Member, IEEE \\ Electrical Department of Engineering \\ Technical University of Catalonia \\ Colom 1- 08222-Terrassa, Spain \\ ${ }^{1}$ Contact Author: alejandro.rolan@upc.edu
}

\begin{abstract}
The aim of this work is to give an approach to the Performance-Oriented Model of Variable-Speed Wind turbines (VSWTs) equipped with permanent magnet synchronous generator (PMSG), and compare it to the component-oriented model. In the present work it will be analyzed how a change in the wind speed affects the power delivered from the generator by means of two approaches: firstly, the component-oriented model of the VSWT-based PMSG is given, i.e. the dynamic equations of the system are shown, and then, it is discussed how to obtain an equivalent transfer function of the whole system. A comparison between both approaches is discussed in this work.
\end{abstract}

\section{INTRODUCTION}

Although the utilization of wind energy has a very long tradition, it was not until the end of the 1990s that wind energy became one of the most important sustainable energy resources, partly because of the increasing price of the oil, security concerns of nuclear power and above all awareness about the climate change. The last issue is one of the main reasons that have lead to a full research into renewal energy sources to provide the electricity demand around the world. Moreover, as wind energy is abundant and it has an inexhaustible potential, it is one of the best technologies today to provide a sustainable supply to the world development.

The use of wind power has growth considerably during lasts years. By the end of 1996 the total capacity of wind power was around $6 \mathrm{GW}$, whereas in 2008 it represented more than $120 \mathrm{GW}$, producing $142 \mathrm{TWh}$ of electricity [1]. It gives an idea of the importance that this renewal energy has nowadays.

The first step to study the integration of wind energy into the electrical grid is to model the whole system properly and simulate it. Nowadays there exist several power system simulation tools, e.g. PSS/E, DigSILENT, SABER or PSCAD/EMTDC (in the present work the simulations has been carried out via PSCAD/EMTDC simulation tool). A comparison between the last two simulation tools is done in [2]. These tools include built-in models of the different components of a wind power plant, which are convenient for load flow and dynamic simulations of power systems. However, it is necessary to develop wind turbine and wind power plant models to analyze the interconnection of these systems to the electrical grid.

Depending on the time frame of the simulation, which is determined by the purpose of the simulation, the level of the detail of the model varies [3]. As a result, the time scale of the simulations will be from few milliseconds to several minutes. With the typical time frame used in large power system stability studies, which approximately $0.1 \mathrm{~s}$ [4], it is not practical to model a wind turbine or a wind power plant that requires a very small time step. Normally, when the complexity of the model increases, it is necessary a smaller integration time. Taking into account this observation, a performance-oriented model [5] for a wind turbine has been proposed as an alternative to a component-oriented model. This approach requires a detailed knowledge of the WTGS as well as the power system analysis to be carried out. For this reason, before developing the performance oriented model of the wind turbine, it is necessary to describe the component oriented model of the WTGS.

This work focuses on the model of VSWTs equipped with PMSG because it is the most promising option for the electricity supply as far as wind energy is concerned. In fact, PMSGs have the windings excited by means of permanent magnets located on the rotor, meaning higher efficiency and less maintenance. What is more, multi-pole PMSGs can be used without a gearbox, which implies a reduction of the weight of the nacelle as well as a reduction in costs.

The rotor aerodynamics, drive train and generator and its control will be modelled, whereas the integration of the system to the electrical grid will not be considered. A comparison between the component-oriented model and the performance-oriented is done in this paper.

\section{SYSTEM DESCRIPTION}

The system analyzed is a VSWT based on a multi-pole PMSG, depicted in Fig. 1. Due to the low generator speed, the rotor shaft is coupled directly to the generator, which means that no gearbox is needed. 


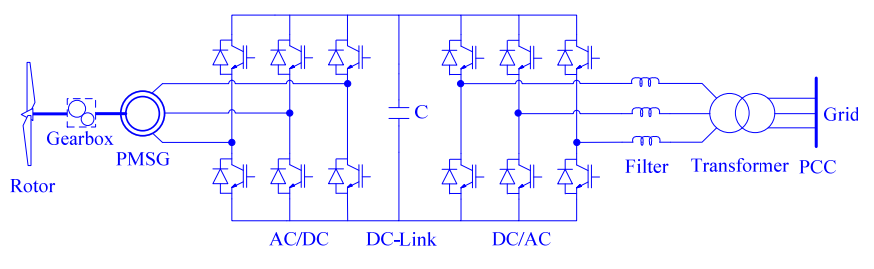

Fig. 1. Electrical scheme of a variable speed wind turbine equipped with a PMSG.

The generator is connected to the grid via a back-to-back converter, which consists of two conventional pulse-width modulated (PWM) voltage source converters (VSCs). The capacitor between the inverter and rectifier permits to decouple the control of the two inverters. The generator-side converter is controlled to regulate the generator speed and the active power supplied by the generator [6][7], whereas the goal of the grid-side converter is to control the reactive power injection to the grid [8][9]. The current is filtered before being supplied to the grid (harmonics must be reduced) and a transformer, which is located between the inverter and the point of common connection (PCC) raise the voltage in order to avoid losses in the transport of the current.

It should be noted that this study is dedicated to model and simulate the system from the wind turbine to the PMSG (with the control of generator-side converter). For this reason, dclink, transformer, grid, rectifier, inverter model and its control as well as pitch angle control will not be considered.

\section{THE COMPONENT-ORIENTED MODEL}

The dynamic equations that describe the model of the each part of the system are described below.

\section{Wind Speed Model}

A complete speed model consists of the sum of four components (constant, ramp, gust and noise) [10]. However, it is usually taken into account a step change in the wind speed in order to analyze if the systems behaves properly due to this change.

\section{Aerodynamic Model}

The aerodynamic model is very well documented in the literature. For example, in [11] one can find that the relation between the power extracted from the wind and the wind speed, which is known as the rotor aerodynamics is

$$
P_{w}=c_{P} \frac{1}{2} \rho A v_{w}^{3}
$$

where $P_{w}$ is the power extracted from the wind (W), $\rho$ is the air density, which is equal to $1.225 \mathrm{~kg} / \mathrm{m}^{3}$ at sea level at temperature $T=288 \mathrm{~K}, c_{P}$ is the power coefficient, $v_{w}$ is the wind speed upstream of the rotor $(\mathrm{m} / \mathrm{s})$ and $A$ is the area swept by the rotor $\left[\mathrm{m}^{2}\right]$ ( $A=\pi R^{2}$, being $R$ the radius of the blade in $\mathrm{m}$ ).

There are four alternatives for modelling the aerodynamic system of a wind turbine, namely the blade element method, $c_{p}$ lookup table, wind speed-mechanical power lookup table and analytical calculation [11]. In this work, the last approach is taken into account. The idea is to approximate the $c_{p}(\lambda, \theta)$ characteristic by the following form

$$
c_{p}(\lambda, \theta)=c_{1}\left(c_{2} \frac{1}{\beta}-c_{3} \theta-c_{4} \theta^{x}-c_{5}\right) e^{-c_{6} \frac{1}{\beta}}
$$

Since the function depends on the wind turbine rotor type, the coefficients $c_{1}-c_{6}$ and $x$ can be different for various WTGS. The proposed coefficients [11] are: $c_{1}=0.5, c_{2}=116$, $c_{3}=0.4, c_{4}=0, c_{5}=5, c_{6}=21\left(x\right.$ is not used because $\left.c_{4}=0\right)$. Additionally, the parameter $\beta$ is also defined in various ways. For example, the parameter $\beta$ in [11] is defined as

$$
\beta=\frac{1}{\frac{1}{\lambda+0.08 \theta}-\frac{0.035}{1+\theta^{3}}}
$$

Where $\theta$ is the pitch angle (rad), which is the angle between the plane of rotation and the blade cross-section chord, and the tip-speed ratio $\lambda$ is defined as

$$
\lambda=\frac{\omega_{t} R}{v_{w}}
$$

where $\omega_{t}$ is the angular velocity of rotor $(\mathrm{rad} / \mathrm{s}), R$ is the rotor radius $(\mathrm{m})$ and $v_{w}$ is the wind speed upstream of the rotor $(\mathrm{m} / \mathrm{s})$.

An example of the $c_{p}(\lambda, \theta)$ characteristics computed taking into account (2-4) and the above parameters $c_{1^{-}} c_{6}$ for a given rotor diameter $R$, rotor speed $\omega_{t}$ and for different values of blade pitch angle $\theta$ is presented in Fig. 2 .

The amount of aerodynamic torque $\left(\tau_{w}\right)$ in $\mathrm{N} \cdot \mathrm{m}$ is given by the ratio between the power extracted from the wind $\left(P_{w}\right)$, in $\mathrm{W}$, and the turbine rotor speed $\left(\omega_{w}\right)$, in $\mathrm{rad} / \mathrm{s}$, as follows

$$
\tau_{w}=\frac{P_{w}}{\omega_{w}}
$$

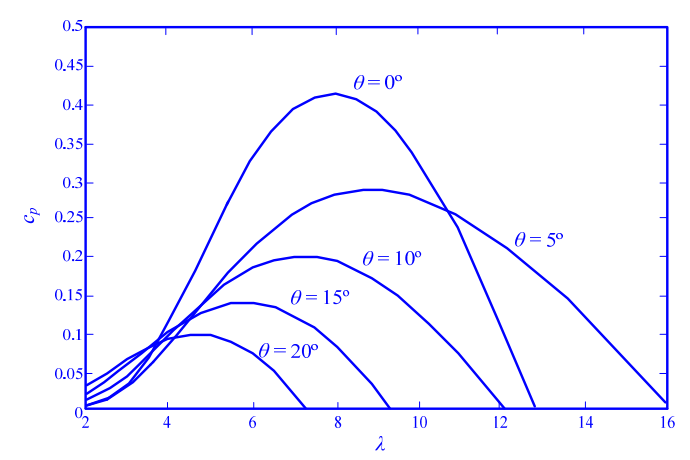

Fig. 2. $c_{p}$ curve for different pitch angles. 


\section{Drive Train Model}

A drive train of a WTGS can be seen as a multi-mass system, consisting of 6-mass, 3-mass, 2-mass or single-mass model [12]. The 6-mass model has six inertias, namely three blade inertias, hub inertia, gearbox inertia and generator inertia. The 3-mass model consists of three inertias, which include the generator rotor, the turbine hub and the blades. In the 2-mass model, the drive train is modelled as two inertias, corresponding to those of turbine and generator.

If a 6-mass or 3-mass model is utilized, the complexity of the model increases, which is not desired in power system stability studies. In fact, only the lower frequency is of interest in such a study. As concluded in [12] a two-mass model is sufficient for representing the dynamics of a turbine drive-train for a power system stability analysis.

Fig. 3 shows a scheme of a mechanical drive train, consisting of two masses. It can be observed the presence of three damping components, namely turbine self damping $D_{t}$ (representing the aerodynamic resistance taking place in the blade), generator self damping $D_{g}$ (which corresponds to mechanical friction and windage) and damping coefficient of the shaft $D_{s}$ (representing the dynamics that occur because of the different speeds between the generator rotor and the turbine shaft). All of damping coefficients are measured in $\mathrm{N} \cdot \mathrm{m} \cdot \mathrm{s} / \mathrm{rad}$.

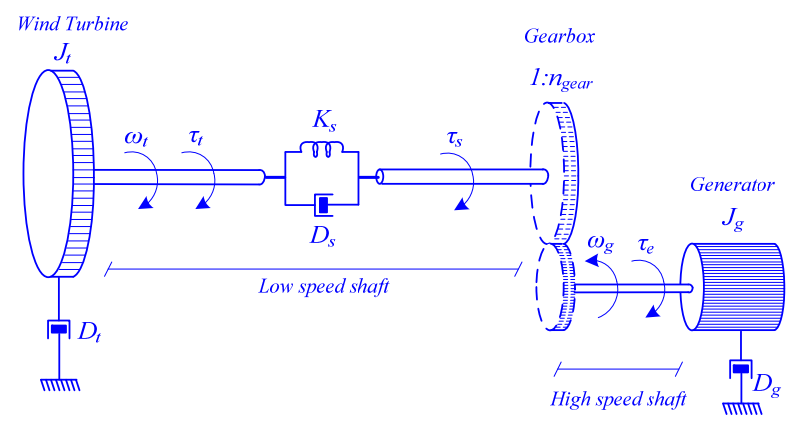

Fig. 3. Scheme of a two-mass drive train.

The mathematical equations of a two-mass drive train model, neglecting the turbine and the generator self dampings, are given by [13]

$$
\begin{aligned}
& J_{t}^{\prime} \frac{d \omega_{t}}{d t}=\tau_{t}^{\prime}-K_{s}^{\prime}\left(\theta_{t}^{\prime}-\theta_{g}\right)-D_{s}^{\prime}\left(\omega_{t}^{\prime}-\omega_{g}\right) \\
& J_{g} \frac{d \omega_{g}}{d t}=-\tau_{e}+K_{s}^{\prime}\left(\theta_{t}^{\prime}-\theta_{g}\right)-D_{s}^{\prime}\left(\omega_{t}^{\prime}-\omega_{g}\right) \\
& \frac{d \theta_{g}}{d t}=\omega_{g} \\
& \frac{d \theta_{t}^{\prime}}{d t}=\omega_{t}^{\prime}
\end{aligned}
$$

where $J_{t}$ and $J_{g}$ are the turbine and the generator inertia constant (both in $\mathrm{kg} \cdot \mathrm{m}^{2}$ ), respectively; $K_{s}$ is the shaft stiffness $(\mathrm{N} \cdot \mathrm{m} / \mathrm{rad}) ; \omega_{t}$ and $\omega_{g}$ are the turbine and the generator rotor speed $(\mathrm{rad} / \mathrm{s})$, respectively; $\tau_{t}$ and $\tau_{e}$ are the torque produced by the rotor and the generator on the low speed and high speed shaft $(\mathrm{N} \cdot \mathrm{m})$, respectively; and $\theta_{t}$ and $\theta_{g}$ denote the turbine and the generator rotor angle ( $\mathrm{rad})$, respectively.

It should be noted that all terms are referred to the high speed shaft (the terms are denoted with the superscript ${ }^{\text {). In }}$ this case, the following transformations are used [13]

$$
\begin{aligned}
& J_{t}^{\prime}=\frac{J_{t}}{n_{\text {gear }}^{2}} ; \quad D_{s}^{\prime}=\frac{D_{s}}{n_{\text {gear }}^{2}} ; \quad K_{s}^{\prime}=\frac{K_{s}}{n_{\text {gear }}^{2}} \\
& \omega_{t}^{\prime}=n_{\text {gear }} \cdot \omega_{t} ; \quad \theta_{t}^{\prime}=n_{\text {gear }} \cdot \theta_{t} ; \quad \tau_{t}^{\prime}=\frac{\tau_{t}}{n_{\text {gear }}}
\end{aligned}
$$

where $n_{\text {gear }}$ is the gearbox ratio.

If the shaft stiffness and mutual damping are neglected, the one-mass model of the drive train is obtained. In this case, there is only a single inertia, representing the sum of the turbine and generator inertias. The mathematical equation of the one-mass model of the drive train is [14]:

$$
\left(\frac{J_{t}}{n_{\text {gear }}^{2}}+J_{g}\right) \frac{d \omega_{g}}{d t}=\tau_{t}-\tau_{e}
$$

It should be noted that the two-mass model takes into account the speed and power fluctuations that appear when non-normal conditions take place.

\section{Generator Model}

The last component that is going to be modelled is the generator, specifically the permanent magnet synchronous generator (PMSG).

When it is desired to obtain a dynamic model of a PMSG one must take into account that only the electrical equations of the stator are considered (because in the rotor there are no windings). It results in a system of three equations, unlike the wound rotor three-phase synchronous machine, in which four equations are needed to be used. However, despite the reduction in the number of equations, it should be noted that it would be very difficult to work with the electrical equations of the PMSG in the static $a b c$ reference-frame, because this equations would depend on the mechanical angle $\theta$ (which is the angle between the rotor position in an instant of time and the $a$-axis), requiring a great deal of simulation time [15]. In order to avoid this problem, these equations are transformed from a static three-phase reference frame to a two-phase rotating reference frame, by means of the Park's transformation [15][16].

Then, the dynamic model of the PMSG is derived from the two-phase rotating reference frame, in which the $d$-axis is aligned with the vector of the flux produced by the permanent magnets and the $q$-axis is $90^{\circ}$ ahead of the $d$-axis with respect to the direction of rotation, as Fig. 4 depicts. This reference frame could be named rotor-oriented reference frame, because the flux created by the permanent magnets rotates at the same speed than that of the rotor. 


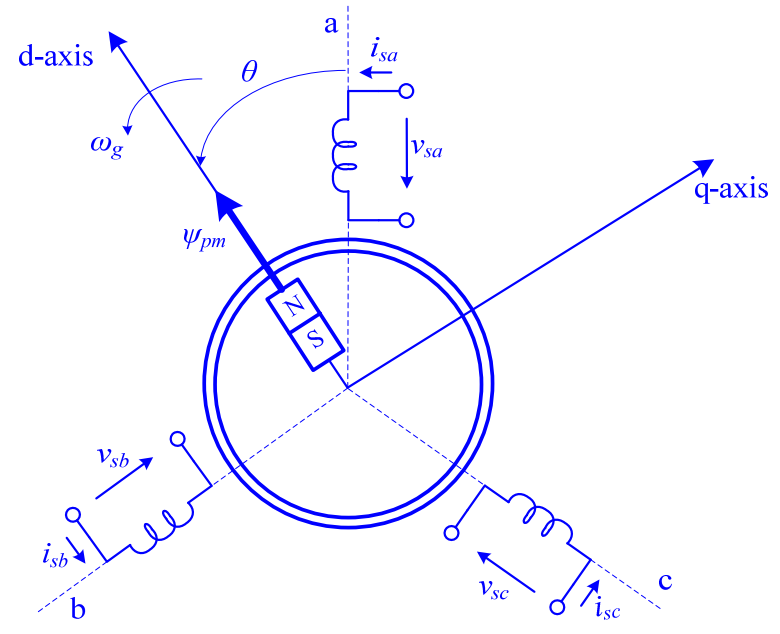

Fig. 4. Scheme of a PMSG, with $a b c$-static reference frame and $d q$-rotating reference frame.

The mathematical model of the PMSG is based on the following assumptions [15][16][17]: the rotor has no damper windings; the machine is symmetric (there exist a separation of $120^{\circ}$ between the stator windings and they have the same inductance); there is no leakage flux in the stator windings, so the leakage inductance of these windings is considered to be zero; the magnetic field has a sinusoidal distribution along the air gap; there is a constant air gap (due to the fact that the permanent magnets are located at the surface of the rotor, so it behaves as a round-rotor machine); magnetic hysteresis and saturation effects are negligible (the magnetic characteristic of the iron is considered to be lineal); the magnetic permeability of the iron is very high, which means that the magnetic reluctance is negligible compared to that of the air gap.

Taking into account these simplifications and considering the rotor-oriented reference frame, the mathematical model of the PMSG can be expressed by the following equations [15]:

$$
\begin{aligned}
& u_{s d}=r_{s} i_{s d}+L_{s d} \frac{d i_{s d}}{d t}-\omega_{e g} L_{s q} i_{s q} \\
& u_{s q}=r_{s} i_{s q}+L_{s q} \frac{d i_{s q}}{d t}+\omega_{e g} L_{s d} i_{s d}+\omega_{e g} \psi_{p m}
\end{aligned}
$$

where subscripts $d$ and $q$ refer to the physical quantities that have been transformed into the $d q$-rotating reference frame; $u_{s d}$ and $u_{s q}$ are the stator voltages $(\mathrm{V}) ; i_{s d}$ and $i_{s q}$ are the stator currents (A); $r_{s}$ is the stator resistance $(\Omega), L_{s d}$ and $L_{s q}$ are the stator inductances $(\mathrm{H})$ in the $d$ and $q$ axis, respectively; $\Psi_{p m}$ is the permanent magnetic flux $(\mathrm{Wb})$; and $\omega_{\text {eg }}$ is the electrical rotating speed of the generator $(\mathrm{rad} / \mathrm{s})$, which is given by:

$$
\omega_{e g}=p \omega_{g}
$$

where $p$ is the number of pole pairs of the generator and $\omega_{g}$ is the rotational speed of the rotor $(\mathrm{rad} / \mathrm{s})$.
To complete the mathematical model of the PMSG the mechanical equation is needed, and it is described by the following electromagnetic torque $\tau_{e}$ equation [15]:

$$
\tau_{e}=1.5 p\left[\left(L_{s d}-L_{s q}\right) i_{s d} i_{s q}+i_{s q} \psi_{p m}\right]
$$

As stated before, the PMSG is assumed to be a round-rotor machine, so inductances in $d$ - and $q$-axis have exactly the same value $\left(L_{s d}=L_{s q}=L_{s}\right)$. Taking into account this simplification, the electrical equations (9), written in the steady-state form [18], and the mechanical equation (11) can be written as follows

$$
\begin{aligned}
& \frac{d i_{s d}}{d t}=\frac{1}{L_{s}}\left(-r_{s} i_{s d}+\omega_{e g} L_{s} i_{s q}+u_{s d}\right) \\
& \frac{d i_{s q}}{d t}=\frac{1}{L_{s}}\left(-r_{s} i_{s q}-\omega_{e g} L_{s} i_{s d}-\omega_{e g} \psi_{p m}+u_{s q}\right) \\
& \tau_{e}=1.5 p i_{s q} \psi_{p m}
\end{aligned}
$$

The active power $P_{\text {gen }}(\mathrm{W})$ and reactive power $Q_{\text {gen }}(\mathrm{VAr})$ of the PMSG are [15]:

$$
\begin{aligned}
& P_{\text {gen }}=1.5\left(u_{s d} i_{s d}+u_{s q} i_{s d}\right) \\
& Q_{\text {gen }}=1.5\left(u_{s q} i_{s d}-u_{s d} i_{s q}\right)
\end{aligned}
$$

\section{The Performance-Oriented Model}

The performance-oriented [5] has arisen as an alternative to the component-oriented model in order to reduce the complexity of the model by reducing the integration time of the simulation, which is usually in the range from 2 to 10 milliseconds.

The idea is to represent the wind turbine as a current source, which depends on various parameters (wind speed, mechanical parameters, etc.). The model basically consists of a set of transfer functions characterizing the wind turbine performance. The final model will consist on a unique transfer function that behaves as if it were a WTGS. In fact, a wind turbine can be described with a second order equivalent transfer function [19], taking into account different simplifications. In [20] it is presented a simplification of the WTGS by obtaining a simplified equivalent model for both the active and reactive power behaviour, once the performance of the wind turbine has been analyzed.

The simplifications that have to be applied in order to obtain a performance-oriented model of the WTGS are as follows [5][20]: wind speed is assumed constant; variablespeed operation in carried out within protection settings; decoupling between power and speed, i.e. pitch angle control aerodynamic representation and mechanical parts can be neglected; the generator dynamics is neglected, so it can be represented as a linear gain (for power system stability, the fast stator flux transient of the generator is not considered): in 
[21], the generator and its converter are simply represented as an algebraic expression that is subjected to power limitation during a fault event; since the generator dynamics is neglected, the dynamic response is given by the response of the power converter, which can be treated as controlled current sources; the protection is simplified to $\mathrm{V} / \mathrm{f}$ protection settings.

All these assumptions lead to a notable reduction in the complexity of the system. Table I [5] shows the differences between the component-oriented model and the performanceoriented model.

TABLE I

Differences Between Performance Model And Component Model

\begin{tabular}{lll}
\hline \hline Feature & Component model & Performance model \\
\hline Generator & Dynamic model & Linear gain \\
Pitch & Pitch control & Not included \\
Mechanical & 2-mass non-linear model & 2-mass linearized \\
system & & model \\
Protection & $\begin{array}{l}\text { Voltage, frequency, over } \\
\text { current and over speed }\end{array}$ & $\begin{array}{l}\text { Voltage and } \\
\text { frequency }\end{array}$ \\
& $\begin{array}{l}\text { Power controller and inner } \\
\text { current loop }\end{array}$ & Power controller \\
Control & $\begin{array}{l}\text { 134+345 data points in look } \\
\text { up table for aerodynamics }\end{array}$ & 37 \\
Parameter count & 62 & 12 \\
& $<1$ ms & \\
Internal states & & $2-10$ ms \\
Time step & &
\end{tabular}

It can be seen that considering the performance-oriented model, significant reduction in model complexity is achieved. However it requires an understanding of the operation of the whole model, as well as the need to know the correct data. Anyway, the simulation time is reduced considerably.

\section{Transfer Function of the VSWT}

The transfer function of the whole system is an $n$-order function in which the input value is the aerodynamic torque and the output value is the active power delivered by the generator. Fig. 5 shows this concept. This transfer function is the result of operating with the transfer functions of the different parts of the system, which will be described below.

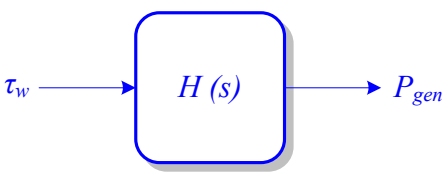

Fig. 5. Wind turbine expressed as a transfer function.

\section{Wind Speed and Aerodynamic Transfer Functions}

There are no transfer functions related to wind speed and aerodynamics because there is no dynamics in the respective equations (1-5). It should be noted from that equations that the aerodynamic torque remains in a constant value provided that the wind speed does not change. So, a step change in wind speed means a step change in aerodynamic torque, which implies a change in active generated power by means of the general transfer function of the system (the input is the wind speed, whereas the output is the aerodynamic torque).

\section{Drive Train Transfer Function}

Taking into account that the 2-mass model drive train equations (6) are linear equations, they can be expressed in the following form

$$
\begin{aligned}
& \omega_{t}=g_{A} \cdot \tau_{w}+g_{B} \cdot \tau_{e} \\
& \omega_{g}=g_{C} \cdot \tau_{w}+g_{D} \cdot \tau_{e}
\end{aligned}
$$

where $g_{A}, g_{B}, g_{C}$ and $g_{D}$ are the following transfer functions

$$
\begin{aligned}
& g_{A}=\left.\frac{\omega_{t}}{\tau_{w}}\right|_{\tau_{e}=0}=\frac{1}{J_{t}^{\prime}+J_{g}} \frac{1}{s} \frac{J_{g} \cdot s^{2}+D_{g}^{\prime} \cdot s+K_{g}^{\prime}}{J \cdot s^{2}+D_{g}^{\prime} \cdot s+K_{g}^{\prime}} \\
& g_{B}=\left.\frac{\omega_{t}}{\tau_{e}}\right|_{\tau_{w}=0}=-\frac{1}{J_{t}^{\prime}+J_{g}} \frac{1}{s} \frac{D_{g}^{\prime} \cdot s+K_{g}^{\prime}}{J \cdot s^{2}+D_{g}^{\prime} \cdot s+K_{g}^{\prime}} \\
& g_{C}=\left.\frac{\omega_{g}}{\tau_{w}}\right|_{\tau_{e}=0}=\frac{1}{J_{t}^{\prime}+J_{g}} \frac{1}{s} \frac{D_{g}^{\prime} \cdot s+K_{g}^{\prime}}{J \cdot s^{2}+D_{g}^{\prime} \cdot s+K_{g}^{\prime}} \\
& g_{D}=\left.\frac{\omega_{g}}{\tau_{e}}\right|_{\tau_{w}=0}=-\frac{1}{J_{t}^{\prime}+J_{g}} \frac{1}{S} \frac{J_{g} \cdot s^{2}+D_{g}^{\prime} \cdot s+K_{g}^{\prime}}{J \cdot s^{2}+D_{g}^{\prime} \cdot s+K_{g}^{\prime}}
\end{aligned}
$$

where

$$
J=\frac{J_{t}^{\prime} \cdot J_{g}}{J_{t}^{\prime}+J_{g}}
$$

As it can be seen, the 2-mass model drive train can be considered as four transfer functions, which means that there are two inputs (torques) and two outputs (speeds).

\section{PMSG Transfer Function}

The PMSG can be expressed as a transfer function in which the input is the generator speed and the output is the active power delivered to the grid. In order to obtain the equivalent transfer function of the PMSG, one must play with the equations (12) and (13), obtaining the expressions that are listed below. Next, by combining this equations together with (14-16) it is possible to obtain the equivalent transfer function of the VSWT-based PMSG, i.e. the performance oriented model.

$$
i_{s d}=\frac{u_{s d}+\omega_{e g} L_{s} i_{s q}}{L_{s} s+r_{s}}
$$




$$
\begin{aligned}
& i_{s q}=\frac{u_{s q}+\omega_{e g} L_{s} i_{s d}-\omega_{e g} \psi_{p m}}{L_{s} s+r_{s}} \\
& =\frac{\left(u_{s d} L_{s}-\omega_{e g} \psi_{p m} L_{s}\right) s+u_{s q} r_{s}}{L_{s}^{2} s^{2}+2 L_{s} r_{s} s-L_{s}^{2} \omega_{e g}^{2}+r_{s}^{2}} \\
& +\frac{\omega_{e g} L_{s} u_{s d}-\omega_{e g} \psi_{p m} r_{s}}{L_{s}^{2} s^{2}+2 L_{s} r_{s} s-L_{s}^{2} \omega_{e g}^{2}+r_{s}^{2}} \\
& \tau_{e}=1.5 p \psi_{p m} i_{s q} \\
& =1.5 p \psi_{p m}\left(\begin{array}{c}
\frac{\left(u_{s d} L_{s}-\omega_{e g} \psi_{p m} L_{s}\right) s+u_{s q} r_{s}}{L_{s}^{2} s^{2}+2 L_{s} r_{s} s-L_{s}^{2} \omega_{e g}^{2}+r_{s}^{2}} \\
+\frac{\omega_{e g} L_{s} u_{s d}-\omega_{e g} \psi_{p m} r_{s}}{L_{s}^{2} s^{2}+2 L_{s} r_{s} s-L_{s}^{2} \omega_{e g}^{2}+r_{s}^{2}}
\end{array}\right) \\
& P_{g e n}=1.5\left(u_{s d} i_{s d}+u_{s q} i_{s d}\right) \\
& =1.5 u_{s d} \frac{u_{s d}+\frac{\omega_{e g} L_{s}\left(\begin{array}{l}
s\left(u_{s q} L_{s}-\omega_{e g} \psi_{p m} L_{s}\right)+ \\
\omega_{e g}\left(L_{s} u_{s d}-\psi_{p m} r_{s}\right)+ \\
u_{s q} r_{s}
\end{array}\right)}{L_{s}^{2} s^{2}+2 L_{s} r_{s} s-L_{s}^{2} \omega_{e g}^{2}+r_{s}^{2}}}{L_{s} s+r_{s}} \\
& +1.5 \frac{\left(\begin{array}{l}
s\left(u_{s q} L_{s}-\omega_{e g} \psi_{p m} L_{s}\right)+ \\
\omega_{e g}\left(L_{s} u_{s d}-\psi_{p m} r_{s}\right)+ \\
u_{s q} r_{s}
\end{array}\right)}{L_{s}^{2} s^{2}+2 L_{s} r_{s} s-L_{s}^{2} \omega_{e g}^{2}+r_{s}^{2}}
\end{aligned}
$$

\section{CONCLuSions}

The equations that explain the behaviour of a variablespeed wind turbine-based permanent magnet synchronous generator have been presented (this approach is named the component-oriented model). The performance-oriented model has been commented by means of the transfer functions of the subsystems in which a wind turbine consists. The idea of this approach is to represent the wind turbine as a current source, which depends on various parameters. The model consists of a set of transfer functions characterizing the wind turbine performance.

Despite the complexity shown by the equations it should be noted that the dynamics that they show are the same as the ones presented by the dynamic models. The idea is to simplify the transfer functions and to obtain an equivalent transfer function that behaves as if it were a wind turbine.

\section{ACKNOWLEDGMENT}

This research work has been developed under the grant ENE2008-06588-C04-03/ALT from the Spanish Ministry of Science and Technology.

\section{REFERENCES}

[1] "Global Wind 2008 Report," Global Wind Energy Council (GWEC), Tech. Rep., March 2009. Available: http://www.gwec.net/

[2] J. A. Fuentes, A. Molina, F. Ruz, E. Gómez, and F. Jiménez, "Wind Turbine Modeling: Comparison of Advanced Tools for Transient Analysis," IEEE Power Engineering Society General Meeting, Tampa, Florida, June 2007, pp. 1-6.

[3] J. O. Tande, "Grid Integration of Wind Farms," Wind Energy, vol. 6, no. 3, pp. 281-295, June 2003.

[4] M. Crow and J. Chen, "The Multirate Method for Simulation of Power System Dynamics," IEEE Transactions on Power Systems, vol. 9, no. 3, pp. 1684-1690, August 1994.

[5] P. Nielsen, G. K. Andersen, K. D. Hagemann, K. Skaug, and J. Bech, "A Performance Oriented Wind Turbine Model for Grid Stability Studies," 12th European Conference on Power Electronics and Applications (EPE), Aalborg, Denmark, September 2007, pp. $1-10$.

[6] M. Chinchilla, S. Arnalte, and J. Burgos, "Control of PermanentMagnet Generators Applied to Variable-Speed Wind-Energy Systems Connected to the Grid," IEEE Transactions on Energy Conversion, vol. 21, no. 1, pp. 130-135, March 2006.

[7] G. Ramtharan, N. Jenkins, and O. Anaya-Lara, "Modelling and Control of Synchronous Generators for Wide-range Variable-speed Wind Turbines," Wind Energy, vol. 10, no. 3, pp. 231-246, March 2007.

[8] A. D. Hansen and G. Michalke, "Modelling and Control of VariableSpeed Multi-pole Permanent Magnet Synchronous Generator Wind Turbine," Wind Energy, vol 11, no. 5, pp. 537-554, May 2008.

[9] S. Achilles and M. Pöller, "Direct Drive Synchronous Machine Models for Stability Assessment of Wind Farms," 4th International Workshop on Large-scale Integration of Wind Power and Transmission Networks for Offshore Wind Farms, Billund, Denmark, October 2003, pp. 1-9.

[10] P. M. Anderson and A. Bose. "Stability Simulation of Wind Turbine Systems," IEEE Transactions on Power Apparatus and Systems, vol. PAS-102, no. 12, December 1983.

[11] S. Heier, Grid Integration of Wind Energy Conversion Systems. 2nd Ed. Chichester: John Wiley \& Sons, 2006.

[12] S. M. Muyeen, Md. H. Ali, R. Takahashi, T. Murata, J. Tamura, Y. Tomaki, A. Sakahara, and E. Sasano, "Comparative study on transient stability analysis of wind turbine generator system using different drive train models," IET Renewable Power Generation, vol. 1, no. 2, pp. 131-141, June 2007.

[13] G. Michalke, "Variable Speed Wind turbines - Modelling, Control, and Impact on Power Systems," Ph.D. Thesis, Dept. Renew. Energ., Technische Universität Darmstadt, Darmstadt, Germany, April 2008.

[14] A. Perdana, "Dynamic Models of Wind Turbines," Ph.D. Thesis, Dept. Energ. And Env., Chalmers University of Technology, Göteborg, Sweden, 2008.

[15] C. Krause, Analysis of Electric Machinery and Drive Systems. 2nd Ed. New York: Wiley-Interscience, 2002.

[16] P. Kundur, Power System Stability and Control. New York: McGraw-Hill, 1994.

[17] J. G. Slootweg, S. W. H. de Haan, H. Polinder, and W. L. Kling, "General Model for Representing Variable Speed Wind Turbines in Power System Dynamics Simulation," IEEE Transactions on Power Systems, vol. 18, no. 1, pp. 144-151, February 2003.

[18] A. Rolán, A. Luna, G. Vázquez, D. Aguilar and G. Azevedo, "Modeling of a Variable Speed Wind Turbine with a Permanent Magnet Synchronous Generator," IEEE International Symposium on Industrial Electronics (ISIE), Seoul, Korea, July 2009, pp. 734-739.

[19] C. Jauch and S. M. Islam, "Identification of a Reduced Order Wind Turbine Transfer Function from the Turbine's Step Response," Australasian Universities Power Engineering Conference (AUPEC), Hobart, Tasmania, Australia, September 2005, pp. 1-4.

[20] J. Soens, J. Driesen, and R. Belmans, "Equivalent Transfer Function for a Variable Speed Wind Turbine in Power System Dynamic Simulations," International Journal of Distributed Energy Sources, vol. 1, no. 2, pp. 111-131, November 2004.

[21] M. Behnke and E. Muljadi, "Reduced Order Dynamic Model for Variable-Speed Wind Turbine with Synchronous Generator and Full Power Conversion Topology," International Conference on Future Power Systems, Amsterdam, The Nederlands, November 2005, pp. $1-6$. 